\title{
Neuroinflammation and cognition across psychiatric conditions
}

\author{
Célia Fourrier (D), ' Gaurav Singhal, ' and Bernhard T. Baune $e^{2 *}$
}

\author{
${ }^{1}$ Discipline of Psychiatry, University of Adelaide, Adelaide, Australia \\ ${ }^{2}$ Department of Psychiatry, Melbourne Medical School, The University of Melbourne, Melbourne, Australia
}

Cognitive impairments reported across psychiatric conditions (ie, major depressive disorder, bipolar disorder, schizophrenia, and posttraumatic stress disorder) strongly impair the quality of life of patients and the recovery of those conditions. There is therefore a great need for consideration for cognitive dysfunction in the management of psychiatric disorders. The redundant pattern of cognitive impairments across such conditions suggests possible shared mechanisms potentially leading to their development. Here, we review for the first time the possible role of inflammation in cognitive dysfunctions across psychiatric disorders. Raised inflammatory processes (microglia activation and elevated cytokine levels) across diagnoses could therefore disrupt neurobiological mechanisms regulating cognition, including Hebbian and homeostatic plasticity, neurogenesis, neurotrophic factor, the HPA axis, and the kynurenine pathway. This redundant association between elevated inflammation and cognitive alterations across psychiatric disorders hence suggests that a cross-disorder approach using pharmacological and nonpharmacological (ie, physical activity and nutrition) anti-inflammatory/immunomodulatory strategies should be considered in the management of cognition in psychiatry.

Received 16 December 2017; Accepted 6 October 2018; First published online 4 February 2019

Key words: Cognition, cognitive dysfunction, cross-diagnoses approach, inflammation, psychiatric disorders.

\section{Introduction}

Cognition has been described as "a suite of interrelated conscious (and unconscious) mental activities, including: pre-attentional sensory gating; attention; learning and memory; problem solving, planning, reasoning and judgment; understanding, knowing and representing; creativity, intuition and insight; 'spontaneous' thought; introspection; as well as mental time travel, selfawareness and meta-cognition (thinking and knowledge about cognition)."1 The range of cognitive impairments reported in psychiatric disorders is wide and redundant across conditions. ${ }^{1}$ These alterations do not only aggravate the course of the disorders but also strongly compromise patients' quality of life and recovery. For example, cognitive symptoms (eg, working memory impairments) in bipolar disorder (BD) or schizophrenia

\footnotetext{
* Address for correspondence: Prof. Bernhard T. Baune, MD, PhD, FRANZCP, Department of Psychiatry and Psychotherapy, University Hospital Münster, University of Münster, Münster, Germany

(Email: bernhard.baune@me.com)

This study is financially supported by a generous research donation of the Fay Fuller Foundation, Adelaide. The funders had no role in preparation of the manuscript or decision to publish.
}

(SCZ) predict the development and the severity of psychotic symptoms, suggesting that they may participate in the development of the diseases. ${ }^{2}$ In addition, symptom severity (ie, intrusive thoughts, nightmares, and flashbacks) in patients with posttraumatic stress disorder (PTSD) is significantly correlated with impairments in attention, learning, memory, executive function, and visuospatial attention. ${ }^{3}$ Cognitive deficits in attention, verbal learning, and verbal memory predict poorer general and psychosocial functioning in BD patients and impair recovery in later stages of the disease. $^{4,5}$ They also negatively affect employment status and occupational functioning in adults with mood disorders or SCZ. ${ }^{4-6}$ There is therefore a great need for consideration for cognitive function in remission and recovery processes in psychiatry, which is emphasized by elevated rates of cognitive impairments after remission $^{7,9,10}$ representing a risk factor for relapse. ${ }^{11}$

A better knowledge of the biological mechanisms underlying cognitive dysfunctions in patients suffering from psychiatric disorders is needed, as they would represent potential therapeutic targets in the management of cognition across psychiatric conditions. Numerous studies have implicated inflammation in the 
development of psychiatric disorders. In particular, it has been suggested that inflammation might underlie core symptoms of the disorders, such as somatic symptoms (eg, fatigue, sleep disturbances, appetite disturbances). ${ }^{12}$ However, there is less evidence linking elevated inflammation to cognitive deficits across psychiatric disorders. ${ }^{12}$ Thus, the purpose of this review is to summarize evidence that supports a role for inflammatory processes in the establishment of cognitive impairments across major depressive disorder (MDD), BD, SCZ, and PTSD.

\section{Biological underpinnings of cognitive function}

Various brain areas and mechanisms participate in the regulation of cognitive function in physiological conditions. In particular, these mechanisms have been extensively described in the hippocampus for learning and memory, whereas the mechanisms underlying other cognitive processes remain understudied. Mechanisms underlying learning and memory processes in the hippocampus encompass changes in neurotransmission at the synapse, namely Hebbian synaptic plasticity (including long term potentiation [LTP] and long term depression [LTD]). ${ }^{13}$ Hippocampal LTP inhibition through blockade or knockout of N-methyl-D-aspartate (NMDA) receptors impairs spatial learning and memory, ${ }^{14}$ whereas enhancing LTP improves learning and memory performances. ${ }^{15}$ In addition, mice exhibiting impaired LTD display behavioral flexibility deficits. ${ }^{16}$ To compensate with prolonged activity changes driven by Hebbian synaptic plasticity, homeostatic mechanisms relying on post-synaptic $\alpha$-amino-3-hydroxy-5-methyl-4isoxazolepropionic acid (AMPA) receptors adjust average synaptic strength to bring the level of post-synaptic response into an appropriate range. ${ }^{17}$ It has been suggested that homeostatic plasticity could be a fundamental mechanism for the dynamic process of memory, providing a link between early and late memory formation processes. ${ }^{18}$ Hippocampal Hebbian plasticity is also tightly interrelated with the formation of new neurons,

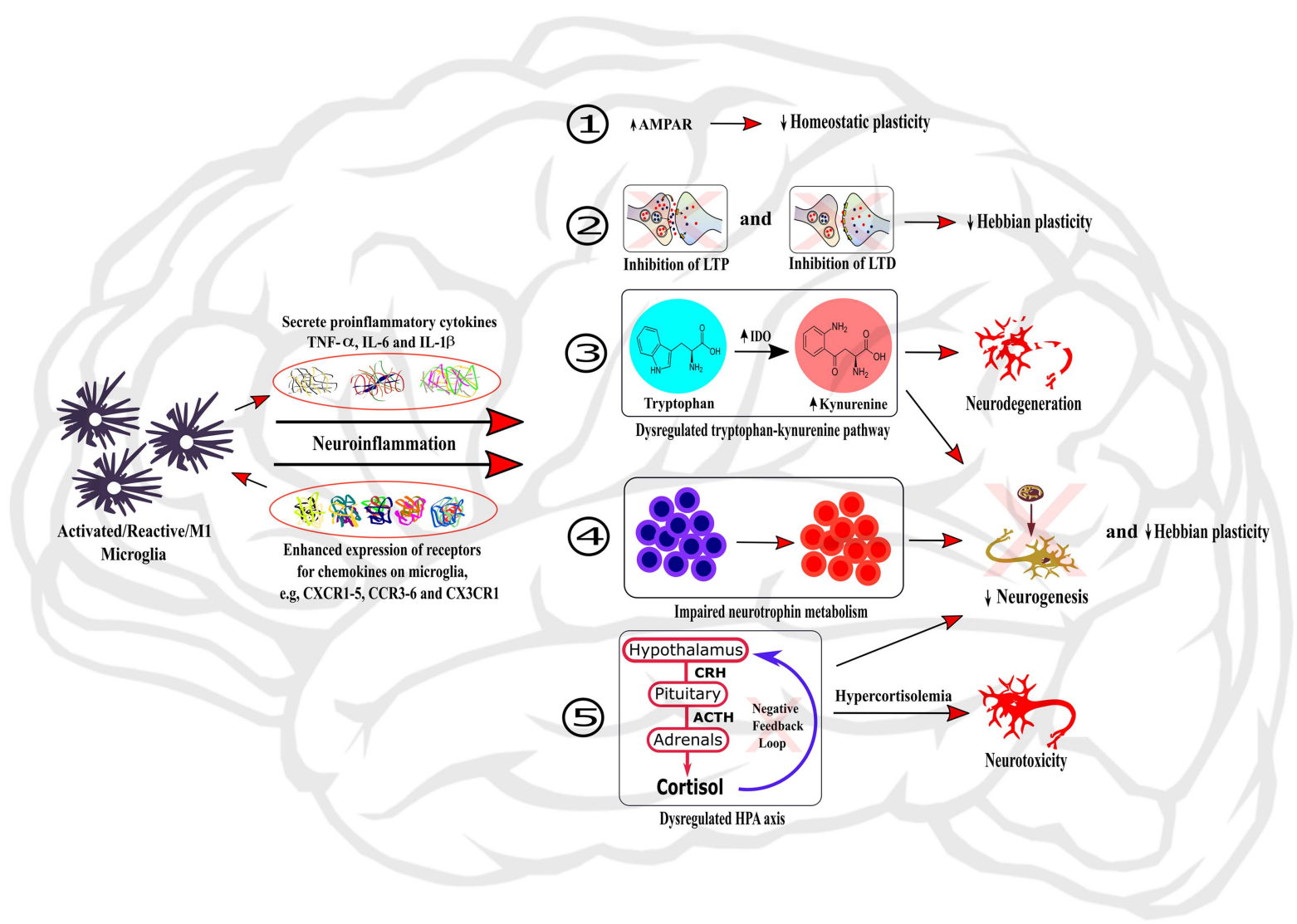

FIGURE 1. Role of neuroinflammation in the dysregulation of neurobiological processes underlying cognition. The activation of microglia within the brain induces neuroinflammation through the secretion of local pro-inflammatory cytokines and the enhanced expression of chemokine receptors on microglia. Neuroinflammation can then induce dysregulations of neurobiological mechanisms regulating cognitive processes by: (1) changing the expression and activity of AMPAR, therefore impairing homeostatic plasticity; (2) inhibiting LTP and LTD processes and hence impairing Hebbian plasticity; (3) dysregulating the tryptophan-kynurenine pathway, subsequently causing neurodegeneration; (4) impairing neurotrophin metabolism; and (5) dysregulating HPA axis, leading to hypercortisolemia and subsequent neurotoxicity. In addition, the processes (3), (4), and (5) also participate to impairment of neurogenesis and Hebbian plasticity processes. 
namely neurogenesis, ${ }^{19,20}$ which also contributes to learning and memory. Indeed, the rate of neurogenesis in rodents was found to be positively correlated to spatial learning and memory performances. ${ }^{21,22}$ In the hippocampus, these key regulatory mechanisms are dependent on the synthesis of neurotrophic factors such as brainderived neurotrophic factor (BDNF) ${ }^{23-26}$ and the activation of various pathways such as the HPA axis ${ }^{27-31}$ and the kynurenine pathway, which are therefore able to modulate learning and memory. ${ }^{32-36}$

\section{Inflammation and cognitive function in physiological conditions: mechanisms}

The relationship between raised levels of inflammation and cognitive changes across inflammation-associated conditions (eg, obesity, rheumatoid arthritis, HIV infection) ${ }^{37-39}$ has suggested a role for inflammation in the regulation of cognition in physiological conditions. The role of peripheral and brain inflammatory mediators (ie, cytokines) in influencing learning and memory was previously reviewed under the name of "cytokine model of cognitive function." ${ }^{40}$ Microglia, the immune cells of the brain, do not only play a role in brain immune function but are also strong regulators of neurological function ${ }^{41}$ (see Figure 1) and cognition in physiological conditions. Indeed, microglia depletion or inhibition in mice negatively impairs learning and memory. ${ }^{42}$ Although microglia can directly modulate cognition, it is noteworthy that they can also perform this role by secreting inflammatory mediators such as cytokines. As previously reviewed, inflammatory cytokines (ie, interleukin-1 $\beta$ [IL-1 $\beta$ ], IL-6, and tumor necrosis factor- $\alpha$ [TNF- $\alpha]$ ) are required for the physiological regulation of memory processes since disrupting their signaling pathway leads to decreased learning and memory. ${ }^{43-46}$ However, the regulatory role of these cytokines on cognition is dose-dependent since overexpression of IL-1 $\beta$ or TNF- $\alpha$ disrupts normal learning and memory in rodents. ${ }^{47,48}$

Recent evidence suggests that disruption of microglia activation alters hippocampus-dependent neuronal plasticity and learning and memory performance in adulthood. ${ }^{49,50}$ Microglial processes continuously interact with synapses in a glutamate-dependent way, ${ }^{51}$ suggesting a role in learning and memory through their impact on synaptic plasticity. In addition, microglia indirectly modulate synaptic plasticity through the production of inflammatory cytokines. ${ }^{52}$ IL-1 $\beta$ and TNF- $\alpha$ are critical in the establishment of synaptic plasticity since their knockout induces impaired $\mathrm{LTP}^{53}$ and $\mathrm{LTD},{ }^{54}$ respectively. Glial-derived TNF- $\alpha$ also strongly regulates homeostatic plasticity by inducing exocytosis of AMPA receptors and inhibiting astrocyte glutamatergic transporters at the synapse. ${ }^{55-57}$ However, the effect of inflammatory cytokines on synaptic plasticity often follows an inverted U-shape since supra-physiological doses of IL-1 $\beta$, IL-6, and TNF- $\alpha$ disrupts normal LTP, ${ }^{58-60}$ possibly linking raised inflammation to cognitive impairments. Similarly to what has been reported for pro-inflammatory cytokines, anti-inflammatory cytokines, such as IL-10, also participate in the regulation of hippocampal synaptic plasticity in a dose-dependent manner. ${ }^{61-63}$ Microglia and pro- and anti-inflammatory cytokines do not only regulate Hebbian and homeostatic plasticity but can also influence brain function through their effects on neurogenesis. During development, microglia coordinate synaptic pruning, ie, the elimination of weak synapses in order to maintain and strengthen functional synapses. ${ }^{64}$ During adulthood, neurogenesis is then highly dependent on the crosstalk between microglia and neurons through the CX3C chemokine receptor 1/CX3C chemokine ligand 1 (CX3CR1/CX3CL1) pathway. ${ }^{65,66}$ Cytokines such as IL-1 $\beta$ and IL- 6 have a dual role on adult neurogenesis in the hippocampus. On one hand, they exert a critical role in the establishment of neurogenesis. ${ }^{67,68}$ On the other hand, their overexpression in the brain negatively affects adult neurogenesis. ${ }^{69,70}$ Similarly, TNF- $\alpha$ may exert a dual role on adult neurogenesis, through a differential effect of its receptors TNF-R1 and TNF-R2, ${ }^{71}$ although the underlying mechanisms remain understudied. Recently, IL-10 was also described as an enhancer of postnatal neurogenesis. ${ }^{72}$

Microglia and inflammatory mediators may also have an indirect effect on cognition-associated biological mechanisms through modulation of neurotrophic factors levels and signaling pathway activation. Although the link between inflammation, cognition, and neurotrophic factors needs further consideration, there is evidence that cytokines can modulate BDNF levels and activity. ${ }^{73}$ In particular, immune stimulation decreases brain BDNF expression and activity, ${ }^{74,75}$ therefore altering synaptic plasticity in the hippocampus. ${ }^{75}$ In mice, BDNF removal from microglia revealed that these cells regulate memory by promoting synapse formation through BDNF signaling. ${ }^{76}$ Along with altering neurotrophic factor activity, cytokine signaling pathways can interact with GC receptor signaling and therefore change GC action. ${ }^{77}$ Inflammatory cytokines can indeed influence the production of all the hormones produced along the hypothalamic-pituitary-adrenal (HPA) axis ${ }^{78}$ and modulate GR function at multiple levels, from expression to translocation and associated signaling pathways. ${ }^{79}$ In addition to their effects on neurotrophic factors and the HPA axis, inflammatory processes can influence kynurenine pathway activation. Pro-inflammatory cytokines induce hippocampal activation of the kynurenineproducing enzyme indoleamine 2,3-dioxygenase (IDO), ${ }^{80,81}$ which participates in the regulation of learning and memory. ${ }^{35,82}$ 


\section{Inflammation and Cognition Across Psychiatric Conditions}

The key role that inflammatory processes play in the regulation of the neurobiological processes underlying cognition in physiological conditions suggests that dysregulations of the immune system could participate in cognitive alterations reported across psychiatric diseases. In agreement with this hypothesis, alterations of neurobiological mechanisms regulating cognition are redundant across disorders (see Table 1). Here, we review the evidence suggesting that inflammatory processes, in particular activated microglia and inflammatory cytokines, play a role in impaired cognitive performance associated with psychiatric disorders through the regulation of the neurobiological processes underlying cognitive function (see Figure 1).

\section{Inflammation and cognition in major depressive disorder}

Levels of inflammatory markers have been found to be associated with cognitive performance in MDD. In patients with MDD, elevated serum levels of TNF- $\alpha$, TNF-R1, and TNF-R2 negatively correlate with performance in executive functioning, attention, learning, working, and declarative memories. ${ }^{83}$ Similarly, elevated plasma levels of C-reactive protein (CRP) and IL-6 are associated with impaired cognitive performance in the domains of attention and executive function and of verbal memory and psychomotor speed, respectively. ${ }^{84-86}$ It is noteworthy that CRP and IL-6 levels are not only associated with cognitive symptoms of depression at baseline but also predict those symptoms at 12 years follow-up, suggesting that inflammation contributes to the progression of MDD rather than to the later stages of the disease. ${ }^{87}$ This relationship appears to be unilateral since cognitive symptoms of depression at baseline are not predictive of inflammatory status at follow-up. Although double-blind, randomized, placebo-controlled trials with anti-inflammatory agents are necessary to establish a causal link between inflammation and cognition in MDD, acute treatment with the cyclooxygenase (COX)-2 inhibitor Celecoxib has been reported to improve cognitive function in an elderly depressed woman with recurrent MDD. ${ }^{88}$

It is noteworthy that individuals with MDD display dysregulations of the kynurenine (KYNA) pathway, which could mediate the relationship between elevated levels of inflammatory markers and cognitive function through its effects on brain plasticity. Elevated levels of inflammatory markers were found to be associated with decreased urinary KYNA in MDD patients. ${ }^{89}$ Interestingly, KYNA/3-hydroxykynurenine (3-HK) was also reported to negatively correlate with hippocampal activity during memory recall, and KYNA/quinolinic acid (QA) correlated with negative specific memory recall and with hippocampal and amygdala volume in MDD patients. ${ }^{90,91}$ It might be hypothesized that the kynurenine pathway could participate in the impairment of cognitive functions observed in MDD by influencing glutamatergic transmission in brain structures associated with cognitive processes. Numerous alterations in glutamatergic synaptic plasticity have indeed been reported in animal models of depression. ${ }^{92}$ Inhibition of microglia activation prevents impairments of both spatial memory and hippocampal LTP in a rodent model of depression. This effect is likely to involve a role of GluR1 phosphorylation. ${ }^{93}$ BDNF is also likely to be a mediator of inflammation-associated cognitive impairments in MDD. In cancer patients with depression, plasma IL-6 levels predict serum BDNF levels, which are significantly associated with short-term memory performance. ${ }^{94}$ In addition, inhibiting TNF- $\alpha$ in a rat model of depression prevents stress-induced cognitive impairments as well as the associated reduction of hippocampal BDNF expression. ${ }^{95}$

\section{Inflammation and cognition in bipolar disorder}

Currently, few studies have reported associations between inflammatory processes and cognitive performances in BD. ${ }^{96}$ In individuals with $\mathrm{BD}$, plasma CRP levels are negatively correlated with immediate memory, language, and attention. ${ }^{97}$ Similarly, elevated levels of

TABLE 1. Main biological mechanisms impairments across psychiatric conditions

\begin{tabular}{lcccc} 
& MDD & BD & SKZ & PTSD \\
\hline Impaired Hebbian plasticity & $+{ }^{(89)}$ & $?$ & $++(148,149)$ & $0 /+{ }^{(154-156)}$ \\
Impaired homeostatic plasticity & $+{ }^{(143)}$ & $?$ & $0 /+{ }^{(150)}$ & $?$ \\
Impaired neurogenesis & $+{ }^{(144,145)}$ & $0 /+{ }^{(100)}$ & $+{ }^{(151)}$ & $+{ }^{(157-159)}$ \\
Decreased peripheral BDNF levels & $+++{ }^{(90,91)}$ & $+++{ }^{(92)}$ & $+++{ }^{(152,153)}$ & $++{ }^{(160-162)}$ \\
Impaired HPA axis activation & $+++{ }^{(146,147)}$ & $+++{ }^{(101,102)}$ & $+++{ }^{(101,146)}$ & $+++(167,168)$ \\
Increased KYNA levels & $++{ }^{(85-87)}$ & $+++{ }^{(103)}$ & $+++(115)$ & $?$ \\
\hline
\end{tabular}

0: essentially absent; 0/+: anecdotal, poorly documented, ambiguous; +: preclinical; ++: clinical; +++: clinical, consistent; ?: not clearly evaluated. Reference numbers are in parentheses. 
IL-1ra and TNF- $\alpha$ are associated with worse memory performances, even during euthymic states. ${ }^{98,99}$ Elevated plasma levels of soluble TNF (sTNF)-RI were also found to be associated with impaired declarative memory in patients with BD. ${ }^{100}$ The only study assessing the relationship between cerebrospinal fluid (CSF) inflammatory markers and cognition in $\mathrm{BD}$ reported a negative association between CSF concentration of the inflammatory biomarker YLK-40 and executive function in those patients. ${ }^{101}$ It is noteworthy that in addition to elevated peripheral inflammation reported in BD patients with cognitive impairments, increased microglial activation was reported in the right hippocampus of BD patients in comparison to healthy controls. ${ }^{102}$ Hence, these studies provide evidence that elevated inflammatory profile is negatively associated with cognitive function in BD.

Various neurobiological systems have been reported to potentially participate in inflammation-associated cognitive impairments in BD. A recent study reported that pro-inflammatory cytokines such as TNF- $\alpha$ decrease white matter integrity in BD individuals, ${ }^{103}$ which could be mediated by alterations in neurogenesis. ${ }^{104}$ Cytokines may also alter cognition by influencing the activity of the HPA axis, subsequently leading to impaired neuroplasticity. Indeed, HPA axis alterations are associated with impaired cognition in individuals with BD. GR insensitivity was reported in $\mathrm{BD},{ }^{105}$ and mifepristone (GR antagonist) treatment for 1 week improves spatial working memory performance in BD. ${ }^{106}$ In addition, a very recent study showed that adult males with $\mathrm{BD}$ display elevated plasma $3-\mathrm{HK} / \mathrm{KYNA}$ ratio, which is significantly associated with poorer declarative memory performances. ${ }^{107}$ Given the role that inflammatory cytokines play in the regulation of the IDO pathway, inflammation may dysregulate this pathway, leading to an imbalance between neuroprotective and neurotoxic metabolites and to a subsequent cognitive impairment in $\mathrm{BD}$ patients. Finally, it is worth mentioning that peripheral BDNF levels, which can also be regulated by inflammation, predict cognitive function in $\mathrm{BD}$. Moreover, the BDNF val66met polymorphism could be a risk factor for cognitive impairment in this disease, ${ }^{96}$ further reinforcing the possible role of BDNF in mediating the effects of inflammation on cognition in BD.

\section{Inflammation and cognition in schizophrenia}

Inflammation has also been extensively reported to be a potential player in the etiology and pathophysiology of SCZ. Epidemiological studies have reported elevated risk of schizophrenia following prenatal or childhood exposure to infection. Infection then mediates peripheral and central inflammatory responses which in turn alter brain development (for a review, see Meyer ${ }^{108}$ ). Similarly to what has been reported in other psychiatric conditions, cognitive impairments associated with schizophrenia are correlated with raised peripheral inflammation. A recent systemic review conducted on SCZ patients reported an association between plasma CRP levels and worse cognitive performance including in the domains of attention, memory, and learning abilities. ${ }^{109}$ Similarly, significant negative associations have been reported between general cognitive function and serum IL-6, sTNF-R1, and IL-1ra levels, ${ }^{98,110}$ and elevated peripheral IL-1 $\beta$ mRNA levels are associated with both impairments in verbal fluency and brain volume reduction in a subgroup of patients with SCZ. ${ }^{111}$ It is noteworthy that an association has also been reported between cognitive impairments and anti-inflammatory cytokines in SCZ, since serum IL-10 levels negatively correlate with cognitive factor (made up of 3 items of the Positive and Negative Syndrome Scale). ${ }^{112,113}$ Particularly, patients who carry the AA allele of the IL10-592 $\mathrm{A} / \mathrm{C}$ polymorphism perform worse in attention, suggesting that this IL-10 allele could contribute to cognitive impairments in SCZ. ${ }^{112}$ Moreover, inflammatory pathways are enriched in mutations associated with cognitive impairments in SCZ patients. ${ }^{114}$ In addition to these studies showing association between inflammation and cognition in SCZ, Müller et $a l^{115}$ reported that decreasing inflammation through anti-inflammatory add-on to risperidone treatment for 5 weeks trends to improve cognition factor in SCZ patients $\left(\mathrm{F}_{1,47}=3.64 ; \mathrm{p}=0.06\right)$.

A longitudinal, double-blind, randomized, placebocontrolled study showed that minocycline add-on to atypical antipsychotic treatment has a beneficial effect on executive functioning (such as working memory, cognitive shifting, and cognitive planning), suggesting that inhibiting microglia activation in patients with SCZ could be a strategy to decrease SCZ-associated cognitive impairments. ${ }^{116}$ This is in agreement with the microglia hypothesis of SCZ, which states that the neuropathology of SCZ is closely associated with elevated microglia activation. ${ }^{117,118}$ Indeed, inflammatory cytokines and free radicals produced by activated microglia in animal models of SCZ lead to decreased neurogenesis, white matter abnormalities, and neuronal degeneration, which may participate in the pathophysiology of the disease.

Similarly to what has been suggested for MDD and BD, inflammation-mediated kynurenine pathway dysfunctions could also participate in cognitive alterations in SCZ, since patients display raised levels of KYNA in the CSF. ${ }^{119}$ This effect could be mediated by changes in glutamatergic neurotransmission. Müller ${ }^{120}$ suggested that elevated inflammation in SCZ may promote the production of the NMDA antagonist KYNA, therefore resulting in a glutamatergic imbalance. The glutamate hypothesis of SCZ suggests that a deficit in glutamatergic transmission in the brain of SCZ patients may lead to 
dopaminergic system dysfunction, which may in turn exacerbate glutamatergic transmission impairments, eventually leading to psychotic and cognitive symptoms. ${ }^{120}$ Decreased glutamatergic neurotransmission may be associated with alterations in synaptic vesicles transportation, which is linked to IL-10 levels in the brain of SCZ patients. ${ }^{112}$

\section{Inflammation and cognition in PTSD}

Currently, the link between inflammatory processes and cognitive impairments remains understudied in the context of PTSD. However, a few recent studies reported elevated levels of inflammatory markers (such as CRP, TNF- $\alpha$, IL- 6 , and IL-1 $\beta$ ) in the plasma of individuals with a diagnosis of PTSD. ${ }^{121-123}$ In a rat model of PTSD, chronic consumption of curcumin, a component with anti-inflammatory properties, impairs the consolidation and reconsolidation of fear memories, suggesting a possible role for inflammation in memory processes. ${ }^{124}$ Interestingly, plasma levels of sTNF-RII are associated with reduced hippocampal volume in Gulf War veterans with current and past PTSD. ${ }^{125}$ Hence, one can hypothesize that inflammation could impair cognition by reducing hippocampal volume via its deleterious effect on neurogenesis or HPA axis activation. ${ }^{126}$ In agreement with this last assumption, the GC receptor antagonist mifepristone improves cognition in Gulf War veterans with PTSD. ${ }^{127}$

\section{Future Directions}

\section{Anti-inflammatory strategies to improve cognition across psychiatric conditions}

The redundant association between inflammatory alterations and cognitive processes across psychiatric disorders suggests that deconstructing psychiatric disorders to account for the heterogeneity across individuals and consider patients' endophenotypes may be promising in the development of novel strategies in the management of cognitive alterations in psychiatry. A cross-disorder approach is in agreement with the Research Domain Criteria (RDoC) initiative led by the National Institute of Mental Health (NIH), which is aimed at developing new ways of classifying mental disorders based on dimensions of both observable behavior and neurobiological measures, in order to develop new and individualized treatments. The evidence reviewed here suggests that the use of anti-inflammatory strategies as adjunctive

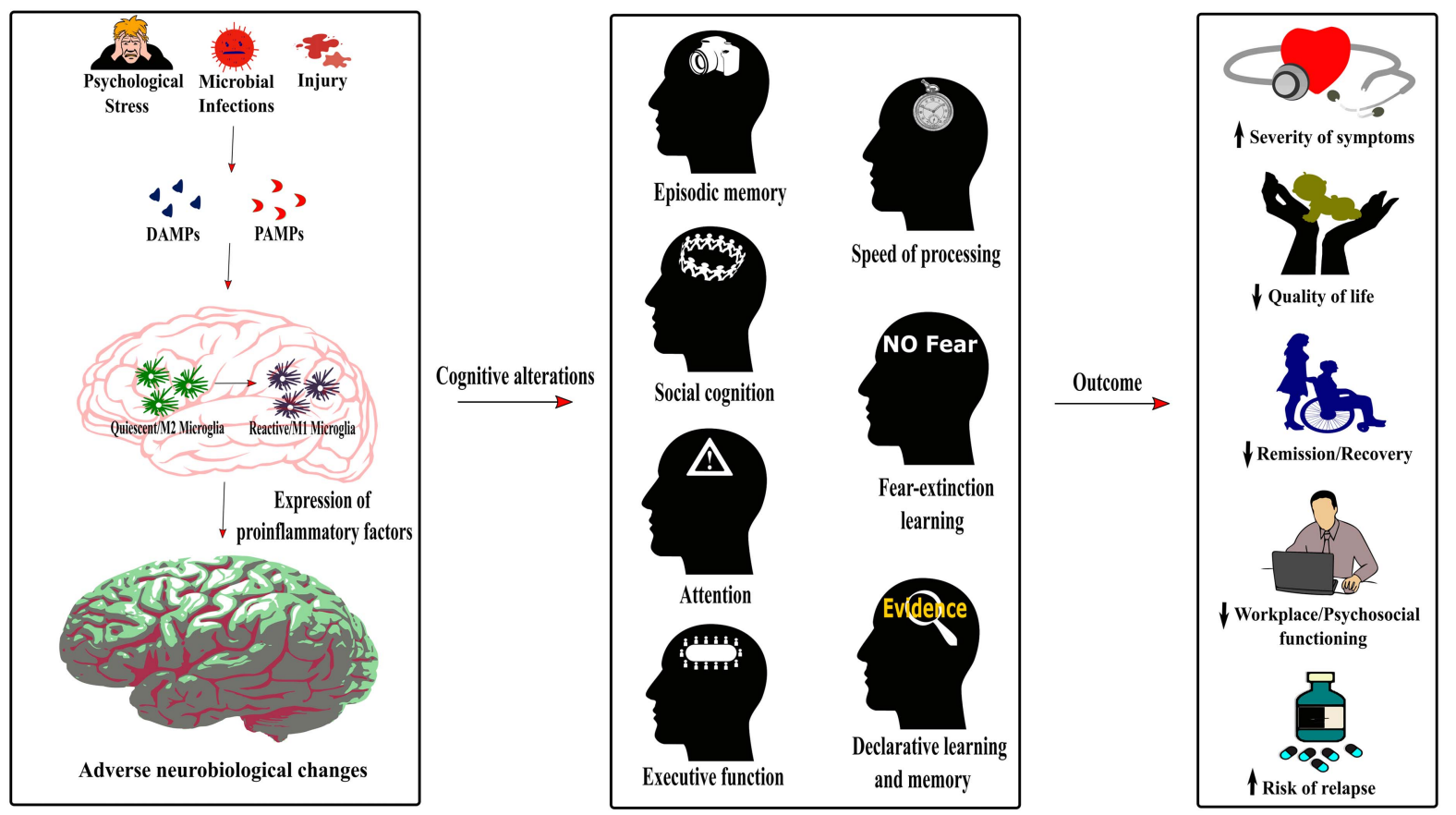

FIGURE 2. Role of neuroinflammation in the cognitive alterations and disease outcomes across psychiatric conditions. The detection of damage-associated molecular patterns (DAMPs) or pathogen-associated molecular patterns (PAMPs) by the immune system following psychological stress, infections or injury induces a switch of the microglia phenotype from a quiescent M2 phenotype to an activated or reactive M1 phenotype. As described in Figure 1, this induces neuroinflammation, therefore dysregulating neurobiological processes underlying cognition. The cognitive alterations resulting from this inflammatory state are redundant across psychiatric conditions and encompass impaired episodic memory, processing speed, social cognition, fear-extinction learning, attention, executive function, and declarative learning and memory. Importantly, this common pattern of cognitive alterations in psychiatric disorders increases the severity of symptoms, decreases the quality of life and workplace and psychosocial functioning of patients, has a negative effect on remission and recovery processes, and increases the risk of relapse. 
therapies across diagnoses could improve the management of cognitive impairments in psychiatry.

\section{Pharmacological approaches}

Nonsteroidal anti-inflammatory drugs (NSAIDs) such as celecoxib (COX-2 inhibitor) are widely used in the treatment of inflammatory conditions (eg, arthritis, multiple sclerosis). In the last decades, they have also been used as effective additional therapies to antidepressant and antipsychotic treatments across psychiatric conditions. ${ }^{128-130}$ However, only a few preliminary data suggest a possible enhancing effect of the antiinflammatory medication celecoxib on cognition in psychiatric disorders. ${ }^{88,115}$ Interestingly, NSAIDs were shown to improve memory in mouse models of neurotoxicity ${ }^{131}$ and Parkinson's disease, ${ }^{132}$ suggesting that their impact on cognition across psychiatric conditions requires further examination.

\section{Nutritional interventions}

Numerous research efforts have reported a beneficial effect of nutrients such as $n-3$ polyunsaturated fatty acids (n-3 PUFAs) and flavonoids on inflammatory processes underlying cognitive dysfunctions. ${ }^{133}$ Indeed, these nutrients and their metabolites have been extensively described to potently reduce microglia activation and cytokine production in inflammatory conditions and improve associated alterations in HPA axis activation, synaptic plasticity, and BDNF production. ${ }^{133-136}$ It is noteworthy that polyphenols (such as flavonoids) and n-3 PUFAs can alleviate cognitive impairments in inflammation-associated conditions such as aging and disorders such as Parkinson's disease and Alzheimer's disease. ${ }^{133,137}$ There is also evidence that they have a beneficial effect on psychiatric disorders, ${ }^{128}$ although it is unclear whether those cognitive improvements are mediated by inflammatory changes.

\section{Physical activity}

Physical activity has been reported to decrease inflammation in both preclinical and clinical models of psychiatric conditions such as MDD. ${ }^{138,139}$ In rodents, exercise does not only enhance anti-inflammatory processes at the cellular level, by changing microglial phenotype, but also at the molecular level by increasing the production of anti-inflammatory cytokines in the brain. ${ }^{138}$ This anti-inflammatory effect of exercise positively influences the activity of the HPA axis as well as neuronal proliferation, neurotrophic factor levels, and the activation of the kynurenine pathway, ${ }^{138}$ which suggests that it could positively impact cognition across psychiatric conditions. In agreement with this hypothesis, moderate physical activity has a beneficial effect on cognition in patients with psychiatric conditions. $^{140-142}$

\section{Conclusion}

The numerous cognitive impairments across major psychiatric disorders highlight a strong need for consideration since they not only affect the quality of life but also the treatment and recovery of patients. However, the mechanisms underlying these deficits are still understudied and must be addressed to allow a better management of the disorders. The common pattern of cognitive impairments across psychiatric conditions suggests shared mechanisms potentially leading to their causation. As described in this review, inflammation could be a shared mechanism underlying the development of cognitive impairments in MDD, BD, SCZ, and PTSD. Indeed, raised inflammatory processes (ie, activated microglia and elevated levels of inflammatory cytokines) can disrupt neurobiological mechanisms regulating cognitive processes (see Figure 2). However, although most studies report associations or correlations between inflammatory biomarkers, cognitive-related biological mechanisms, and cognitive performance, causal evidence is still strongly lacking. Few studies have evaluated the role of inflammation in cognitive alterations in other psychiatric conditions such as autism or attention deficit hyperactivity disorder, but one may hypothesize that the underlying mechanisms could be similar given the shared pattern of cognitive alteration across psychiatric conditions. $^{1}$

\section{Disclosures}

Célia Fourrier and Gaurav Singha have nothing to disclose. Bernhard Baune has the following disclosure: Grants from Fay Fuller Foundation, during the conduct of the study.

\section{REFERENCES:}

1. Millan MJ, Agid Y, Brune M, et al. Cognitive dysfunction in psychiatric disorders: characteristics, causes and the quest for improved therapy. Nat Rev Drug Discov. 2012; 11(2): 141-168.

2. Jenkins LM, Bodapati AS, Sharma RP, Rosen C. Working memory predicts presence of auditory verbal hallucinations in schizophrenia and bipolar disorder with psychosis. J Clin Exp Neuropsychol. 2018; 40(1): 84-94.

3. Qureshi SU, Long ME, Bradshaw MR, et al. Does PTSD impair cognition beyond the effect of trauma? J Neuropsychiatry Clin Neurosci. 2011; 23(1): 16-28.

4. Baune BT, Li X, Beblo T. Short- and long-term relationships between neurocognitive performance and general function in bipolar disorder. J Clin Exp Neuropsychol. 2013; 35(7): 759-774. 
5. Andreou C, Bozikas VP. The predictive significance of neurocognitive factors for functional outcome in bipolar disorder Curr Opin Psychiatry. 2013; 26(1): 54-59.

6. Tse S, Chan S, Ng KL, Yatham LN. Meta-analysis of predictors of favorable employment outcomes among individuals with bipolar disorder. Bipolar Disord. 2014; 16(3): 217-229.

7. Kar SK, Jain M. Current understandings about cognition and the neurobiological correlates in schizophrenia. J Neurosci Rural Pract. 2016; 7(3): 412-418.

8. Baune BT, Renger L. Pharmacological and non-pharmacological interventions to improve cognitive dysfunction and functional ability in clinical depression-a systematic review. Psychiatry Res. 2014; 219(1): 25-50.

9. Rock PL, Roiser JP, Riedel WJ, Blackwell AD. Cognitive impairment in depression: a systematic review and meta-analysis. Psychol Med. 2014; 44(10): 2029-2040.

10. Soni A, Singh P, Shah R, Bagotia S. Impact of cognition and clinical factors on functional outcome in patients with bipolar disorder. East Asian Arch Psychiatry. 2017; 27(1): 26-34.

11. Majer M, Ising M, Kunzel H, et al. Impaired divided attention predicts delayed response and risk to relapse in subjects with depressive disorders. Psychol Med. 2004; 34(8): 1453-1463.

12. Dooley LN, Kuhlman KR, Robles TF, Eisenberger NI, Craske MG, Bower JE. The role of inflammation in core features of depression: insights from paradigms using exogenously-induced inflammation. Neurosci Biobehav Rev. 2018; 94: 219-237.

13. Citri A, Malenka RC. Synaptic plasticity: multiple forms, functions, and mechanisms. Neuropsychopharmacology. 2008; 33 (1): 18-41.

14. Martin SJ, Morris RG. New life in an old idea: the synaptic plasticity and memory hypothesis revisited. Hippocampus. 2002; 12(5): 609-636.

15. Perusini JN, Cajigas SA, Cohensedgh O, et al. Optogenetic stimulation of dentate gyrus engrams restores memory in Alzheimer's disease mice. Hippocampus. 2017; 27(10): 1110-1122.

16. Dong Z, Bai Y, Wu X, et al. Hippocampal long-term depression mediates spatial reversal learning in the Morris water maze. Neuropharmacology. 2013; 64: 65-73.

17. Fernandes D, Carvalho AL. Mechanisms of homeostatic plasticity in the excitatory synapse. J Neurochem. 2016; 139(6): 973-996.

18. Tetzlaff C, Kolodziejski C, Timme M, Tsodyks M, Worgotter F. Synaptic scaling enables dynamically distinct short- and long-term memory formation. PLoS Comput Biol. 2013; 9(10): e1003307.

19. Bruel-Jungerman E, Davis S, Rampon C, Laroche S. Long-term potentiation enhances neurogenesis in the adult dentate gyrus. $J$ Neurosci. 2006; 26(22): 5888-5893.

20. Nissant A, Bardy C, Katagiri H, Murray K, Lledo PM. Adult neurogenesis promotes synaptic plasticity in the olfactory bulb. Nat Neurosci. 2009; 12(6): 728-730.

21. Hollands C, Tobin MK, Hsu M, et al. Depletion of adult neurogenesis exacerbates cognitive deficits in Alzheimer's disease by compromising hippocampal inhibition. Mol Neurodegener. 2017; 12(1): 64

22. Richetin K, Leclerc C, Toni N, et al. Genetic manipulation of adultborn hippocampal neurons rescues memory in a mouse model of Alzheimer's disease. Brain. 2015; 138(Pt 2): 440-455.

23. Leal G, Bramham CR, Duarte CB. BDNF and hippocampal synaptic plasticity. Vitam Horm. 2017; 104: 153-195.

24. Carvalho AL, Caldeira MV, Santos SD, Duarte CB. Role of the brain-derived neurotrophic factor at glutamatergic synapses. $\mathrm{Br} \mathrm{J}$ Pharmacol. 2008; 153(Suppl 1): S310-324.

25. Scharfman H, Goodman J, Macleod A, Phani S, Antonelli C, Croll $\mathrm{S}$. Increased neurogenesis and the ectopic granule cells after intrahippocampal BDNF infusion in adult rats. Exp Neurol. 2005; 192(2): $348-356$.
26. Aarse J, Herlitze S, Manahan-Vaughan D. The requirement of BDNF for hippocampal synaptic plasticity is experiencedependent. Hippocampus. 2016; 26(6): 739-751.

27. Kino T. Stress, glucocorticoid hormones, and hippocampal neural progenitor cells: implications to mood disorders. Front Physiol. 2015; 6: 230 .

28. Groc L, Choquet D, Chaouloff F. The stress hormone corticosterone conditions AMPAR surface trafficking and synaptic potentiation. Nat Neurosci. 2008; 11(8): 868-870.

29. Joëls M, Karst H. Corticosteroid effects on calcium signaling in limbic neurons. Cell Calcium. 2012; 51(3-4): 277-283.

30. Howland JG, Cazakoff BN. Effects of acute stress and GluN2Bcontaining NMDA receptor antagonism on object and object-place recognition memory. Neurobiol Learn Mem. 2010; 93(2): 261-267.

31. Pocivavsek A, Wu HQ, Potter MC, Elmer GI, Pellicciari R, Schwarcz R. Fluctuations in endogenous kynurenic acid control hippocampal glutamate and memory. Neuropsychopharmacology. 2011; 36(11): 2357-2367.

32. de Quervain D, Schwabe L, Roozendaal B. Stress, glucocorticoids and memory: implications for treating fear-related disorders. Nat Rev Neurosci. 2017; 18(1): 7-19.

33. Allison DJ, Ditor DS. The common inflammatory etiology of depression and cognitive impairment: a therapeutic target. J Neuroinflammation. 2014; 11: 151.

34. Alexander KS, Wu HQ, Schwarcz R, Bruno JP. Acute elevations of brain kynurenic acid impair cognitive flexibility: normalization by the alpha7 positive modulator galantamine. Psychopharmacology (Berl). 2012; 220(3): 627-637.

35. Heisler JM, O'Connor JC. Indoleamine 2,3-dioxygenasedependent neurotoxic kynurenine metabolism mediates inflammation-induced deficit in recognition memory. Brain Behav Immun. 2015; 50: 115-124.

36. Pocivavsek A, Wu HQ, Elmer GI, Bruno JP, Schwarcz R. Pre- and postnatal exposure to kynurenine causes cognitive deficits in adulthood. Eur J Neurosci. 2012; 35(10): 1605-1612.

37. Castanon N, Lasselin J, Capuron L. Neuropsychiatric comorbidity in obesity: role of inflammatory processes. Front Endocrinol (Lausanne). 2014; 5: 74 .

38. Joaquim AF, Appenzeller S. Neuropsychiatric manifestations in rheumatoid arthritis. Autoimmun Rev. 2015; 14(12): 1116-1122.

39. Saylor D, Dickens AM, Sacktor N, et al. HIV-associated neurocognitive disorder-pathogenesis and prospects for treatment. Nat Rev Neurol. 2016; 12(5): 309.

40. McAfoose J, Baune BT. Evidence for a cytokine model of cognitive function. Neurosci Biobehav Rev. 2009; 33(3): 355-366.

41. Singhal G, Baune BT. Microglia: an interface between the loss of neuroplasticity and depression. Front Cell Neurosci. 2017; 11: 270

42. Torres L, Danver J, Ji K, et al. Dynamic microglial modulation of spatial learning and social behavior. Brain Behav Immun. 2016; 55: $6-16$.

43. Baune BT, Wiede F, Braun A, Golledge J, Arolt V, Koerner H. Cognitive dysfunction in mice deficient for TNF- and its receptors. Am J Med Genet B. 2008; 147b(7): 1056-1064.

44. Camara ML, Corrigan F, Jaehne EJ, et al. TNF-alpha and its receptors modulate complex behaviours and neurotrophins in transgenic mice. Psychoneuroendocrinology. 2013; 38(12): 31023114 .

45. Goshen I, Kreisel T, Ounallah-Saad H, et al. A dual role for interleukin-1 in hippocampal-dependent memory processes. Psychoneuroendocrinology. 2007; 32(8-10): 1106-1115.

46. Hryniewicz A, Bialuk I, Kamiński KA, Winnicka MM. Impairment of recognition memory in interleukin (IL)-6 knock-out mice. Eur J Pharmacol. 2007; 577(1-3): 219-220.

47. Barrientos RM, Higgins EA, Sprunger DB, Watkins LR, Rudy JW, Maier SF. Memory for context is impaired by a post context 
exposure injection of interleukin-1 beta into dorsal hippocampus. Behav Brain Res. 2002; 134(1-2): 291-298.

48. Fiore M, Angelucci F, Alleva E, Branchi I, Probert L, Aloe L. Learning performances, brain NGF distribution and NPY levels in transgenic mice expressing TNF-alpha. Behavioural Brain Research. 2000; 112 (1-2): 165-175.

49. Maggi L, Scianni M, Branchi I, D’Andrea I, Lauro C, Limatola C. CX(3)CR1 deficiency alters hippocampal-dependent plasticity phenomena blunting the effects of enriched environment. Front Cell Neurosci. 2011; 5: 22.

50. Rogers JT, Morganti JM, Bachstetter AD, et al. CX3CR1 deficiency leads to impairment of hippocampal cognitive function and synaptic plasticity. J Neurosci. 2011; 31(45): 16241-16250.

51. Eyo UB, Peng J, Swiatkowski P, Mukherjee A, Bispo A, Wu LJ. Neuronal hyperactivity recruits microglial processes via neuronal NMDA receptors and microglial P2Y12 receptors after status epilepticus. J Neurosci. 2014; 34(32): 10528-10540.

52. Pickering M, O'Connor JJ. Pro-inflammatory cytokines and their effects in the dentate gyrus. Prog Brain Res. 2007; 163: 339-354.

53. Avital A, Goshen I, Kamsler A, et al. Impaired interleukin-1 signaling is associated with deficits in hippocampal memory processes and neural plasticity. Hippocampus. 2003; 13(7): 826-834.

54. Albensi BC, Mattson MP. Evidence for the involvement of TNF and NF-kappaB in hippocampal synaptic plasticity. Synapse. 2000; 35 (2): 151-159.

55. Stellwagen D, Beattie EC, Seo JY, Malenka RC. Differential regulation of AMPA receptor and GABA receptor trafficking by tumor necrosis factor-alpha. JNeurosci. 2005; 25(12): 3219-3228.

56. Stellwagen D, Malenka RC. Synaptic scaling mediated by glial TNF-alpha. Nature. 2006; 440(7087): 1054-1059.

57. Beattie EC, Stellwagen D, Morishita W, et al. Control of synaptic strength by glial TNFalpha. Science. 2002; 295(5563): 2282-2285.

58. Murray CA, Lynch MA. Evidence that increased hippocampal expression of the cytokine interleukin-1 beta is a common trigger for age- and stress-induced impairments in long-term potentiation. J Neurosci. 1998; 18(8): 2974-2981.

59. Tancredi V, D'Antuono M, Cafe C, et al. The inhibitory effects of interleukin- 6 on synaptic plasticity in the rat hippocampus are associated with an inhibition of mitogen-activated protein kinase ERK. J Neurochem. 2000; 75(2): 634-643.

60. Cumiskey D, Butler MP, Moynagh PN, O'Connor J J. Evidence for a role for the group I metabotropic glutamate receptor in the inhibitory effect of tumor necrosis factor-alpha on long-term potentiation. Brain Res. 2007; 1136(1): 13-19.

61. Kelly A, Lynch A, Vereker E, et al. The anti-inflammatory cytokine, interleukin (IL)-10, blocks the inhibitory effect of IL-1 beta on long term potentiation. A role for JNK. J Biol Chem. 2001; 276(49): 45564-45572.

62. Lynch AM, Walsh C, Delaney A, Nolan Y, Campbell VA, Lynch MA. Lipopolysaccharide-induced increase in signalling in hippocampus is abrogated by IL-10-a role for IL-1 beta? J Neurochem. 2004; 88 (3): 635-646.

63. Almolda B, de Labra C, Barrera I, et al. Alterations in microglial phenotype and hippocampal neuronal function in transgenic mice with astrocyte-targeted production of interleukin-10. Brain Behav Immun. 2015; 45: 80-97.

64. Hua JY, Smith SJ. Neural activity and the dynamics of central nervous system development. Nat Neurosci. 2004; 7(4): 327-332.

65. Delpech JC, Madore C, Nadjar A, Joffre C, Wohleb ES, Laye S. Microglia in neuronal plasticity: influence of stress. Neuropharmacology. 2015; 96(Pt A): 19-28.

66. de Miranda AS, Zhang CJ, Katsumoto A, Teixeira AL Hippocampal adult neurogenesis: does the immune system matter? J Neurol Sci. 2017; 372: 482-495.
67. Meng C, Zhang JC, Shi RL, Zhang SH, Yuan SY. Inhibition of interleukin-6 abolishes the promoting effects of pair housing on post-stroke neurogenesis. Neuroscience. 2015; 307: 160-170.

68. Spulber S, Oprica M, Bartfai T, Winblad B, Schultzberg M. Blunted neurogenesis and gliosis due to transgenic overexpression of human soluble IL-1ra in the mouse. Eur J Neurosci. 2008; 27(3): 549-558.

69. Vallieres L, Campbell IL, Gage FH, Sawchenko PE. Reduced hippocampal neurogenesis in adult transgenic mice with chronic astrocytic production of interleukin-6. J Neurosci. 2002; 22(2): 486-492.

70. Wu MD, Hein AM, Moravan MJ, Shaftel SS, Olschowka JA, O'Banion MK. Adult murine hippocampal neurogenesis is inhibited by sustained IL-1beta and not rescued by voluntary running. Brain Behav Immun. 2012; 26(2): 292-300.

71. Chen Z, Palmer TD. Differential roles of TNFR1 and TNFR2 signaling in adult hippocampal neurogenesis. Brain Behav Immun. 2013; 30: 45-53.

72. Pereira L, Font-Nieves M, Van den Haute C, Baekelandt V, Planas AM, Pozas E. IL-10 regulates adult neurogenesis by modulating ERK and STAT3 activity. Front Cell Neurosci. 2015; 9: 57 .

73. Calabrese F, Rossetti AC, Racagni G, Gass P, Riva MA, Molteni R. Brain-derived neurotrophic factor: a bridge between inflammation and neuroplasticity. Front Cell Neurosci. 2014; 8: 430.

74. Gonzalez P, Machado I, Vilcaes A, et al. Molecular mechanisms involved in interleukin 1-beta (IL-1beta)-induced memory impairment. Modulation by alpha-melanocyte-stimulating hormone (alpha-MSH). Brain Behav Immun. 2013; 34: 141-150.

75. Tong L, Prieto GA, Kramar EA, et al. Brain-derived neurotrophic factor-dependent synaptic plasticity is suppressed by interleukin1beta via p38 mitogen-activated protein kinase. J Neurosci. 2012; 32(49): 17714-17724.

76. Parkhurst CN, Yang G, Ninan I, et al. Microglia promote learning dependent synapse formation through brain-derived neurotrophic factor. Cell. 2013; 155(7): 1596-1609.

77. Pace TW, Hu F, Miller AH. Cytokine-effects on glucocorticoid receptor function: relevance to glucocorticoid resistance and the pathophysiology and treatment of major depression. Brain Behav Immun. 2007; 21(1): 9-19.

78. Silverman MN, Sternberg EM. Glucocorticoid regulation of inflammation and its functional correlates: from HPA axis to glucocorticoid receptor dysfunction. Ann N Y Acad Sci. 2012; 1261(1): 55-63.

79. Pace TW, Miller AH. Cytokines and glucocorticoid receptor signaling. Relevance to major depression. Ann N Y Acad Sci. 2009; 1179(1): 86-105.

80. Andre C, O'Connor JC, Kelley KW, Lestage J, Dantzer R, Castanon N. Spatio-temporal differences in the profile of murine brain expression of proinflammatory cytokines and indoleamine 2,3dioxygenase in response to peripheral lipopolysaccharide administration. J Neuroimmunol. 2008; 200(1-2): 90-99.

81. O'Connor JC, Andre C, Wang Y, et al. Interferon-gamma and tumor necrosis factor-alpha mediate the upregulation of indoleamine 2,3-dioxygenase and the induction of depressive-like behavior in mice in response to bacillus Calmette-Guerin. $J$ Neurosci. 2009; 29(13): 4200-4209.

82. Too LK, Li KM, Suarna C, et al. Deletion of TDO2, IDO-1 and IDO-2 differentially affects mouse behavior and cognitive function. Behav Brain Res. 2016; 312: 102-117.

83. Bobinska K, Galecka E, Szemraj J, Galecki P, Talarowska M. Is there a link between TNF gene expression and cognitive deficits in depression? Acta Biochim Pol. 2017; 64(1): 65-73.

84. Chang HH, Lee IH, Gean PW, et al. Treatment response and cognitive impairment in major depression: association 
with C-reactive protein. Brain Behav Immun. 2012; 26(1): 90-95.

85. Grassi-Oliveira R, Bauer ME, Pezzi JC, Teixeira AL, Brietzke E. Interleukin-6 and verbal memory in recurrent major depressive disorder. Neuro Endocrinol Lett. 2011; 32(4): 540-544.

86. Goldsmith DR, Haroon E, Woolwine BJ, et al. Inflammatory markers are associated with decreased psychomotor speed in patients with major depressive disorder. Brain Behav Immun. 2016; 56: 281-288.

87. Gimeno D, Kivimaki M, Brunner EJ, et al. Associations of C-reactive protein and interleukin- 6 with cognitive symptoms of depression: 12-year follow-up of the Whitehall II study. Psychol Med. 2009; 39(3): 413-423.

88. Chen CY, Tzeng NS, Chen YC. Maintenance therapy of celecoxib for major depression with mimicking neuropsychological dysfunction. Gen Hosp Psychiatry. 2010; 32(6): e647-649.

89. Peacock BN, Scheiderer DJ, Kellermann GH. Biomolecular aspects of depression: a retrospective analysis. Compr Psychiatry. 2017; 73: 168-180.

90. Young KD, Drevets WC, Dantzer R, Teague TK, Bodurka J, Savitz J. Kynurenine pathway metabolites are associated with hippocampal activity during autobiographical memory recall in patients with depression. Brain Behav Immun. 2016; 56: 335-342.

91. Savitz J, Drevets WC, Smith CM, et al. Putative neuroprotective and neurotoxic kynurenine pathway metabolites are associated with hippocampal and amygdalar volumes in subjects with major depressive disorder. Neuropsychopharmacology. 2015; 40(2): 463-471.

92. Mahati K, Bhagya V, Christofer T, Sneha A, Shankaranarayana Rao BS. Enriched environment ameliorates depression-induced cognitive deficits and restores abnormal hippocampal synaptic plasticity. Neurobiol Learn Mem. 2016; 134 Pt B: 379-391.

93. Liu M, Li J, Dai P, et al. Microglia activation regulates GluR1 phosphorylation in chronic unpredictable stress-induced cognitive dysfunction. Stress. 2015; 18(1): 96-106.

94. Jehn CF, Becker B, Flath B, et al. Neurocognitive function, brainderived neurotrophic factor (BDNF) and IL-6 levels in cancer patients with depression. J Neuroimmunol. 2015; 287: 88-92.

95. Sahin TD, Karson A, Balci F, Yazir Y, Bayramgurler D, Utkan T. TNF-alpha inhibition prevents cognitive decline and maintains hippocampal BDNF levels in the unpredictable chronic mild stress rat model of depression. Behav Brain Res. 2015; 292: 233-240.

96. Bauer IE, Pascoe MC, Wollenhaupt-Aguiar B, Kapczinski F, Soares JC. Inflammatory mediators of cognitive impairment in bipolar disorder. J Psychiatr Res. 2014; 56: 18-27.

97. Dickerson F, Stallings C, Origoni A, Vaughan C, Khushalani S, Yolken R. Elevated C-reactive protein and cognitive deficits in individuals with bipolar disorder. J Affect Disord. 2013; 150(2): 456-459.

98. Hope S, Hoseth E, Dieset I, et al. Inflammatory markers are associated with general cognitive abilities in schizophrenia and bipolar disorder patients and healthy controls. Schizophr Res. 2015; 165(2-3): 188-194.

99. Doganavsargil-Baysal O, Cinemre B, Aksoy UM, et al. Levels of TNF-alpha, soluble TNF receptors (sTNFR1, sTNFR2), and cognition in bipolar disorder. Hum Psychopharmacol. 2013; 28 (2): 160-167.

100. Hoseth EZ, Westlye LT, Hope S, et al. Association between cytokine levels, verbal memory and hippocampus volume in psychotic disorders and healthy controls. Acta Psychiatr Scand. 2016; 133(1): 53-62.

101. Rolstad S, Jakobsson J, Sellgren C, et al. CSF neuroinflammatory biomarkers in bipolar disorder are associated with cognitive impairment. Eur Neuropsychopharmacol. 2015; 25(8): 1091-1098.

102. Haarman BC, Riemersma-Van der Lek RF, de Groot JC, et al. Neuroinflammation in bipolar disorder-a [(11)C]-(R)-PK11195 positron emission tomography study. Brain Behav Immun. 2014; 40: 219-225.

103. Benedetti F, Poletti S, Hoogenboezem TA, et al. Inflammatory cytokines influence measures of white matter integrity in bipolar disorder. J Affect Disord. 2016; 202: 1-9.

104. Czeh B, Lucassen PJ. What causes the hippocampal volume decrease in depression? Are neurogenesis, glial changes and apoptosis implicated? Eur Arch Psychiatry Clin Neurosci. 2007; 257(5): 250-260.

105. Fries GR, Vasconcelos-Moreno MP, Gubert C, et al. Hypothalamicpituitary-adrenal axis dysfunction and illness progression in bipolar disorder. Int J Neuropsychopharmacol. 2014; 18(1): pyu043.

106. Watson S, Gallagher P, Porter RJ, et al. A randomized trial to examine the effect of mifepristone on neuropsychological performance and mood in patients with bipolar depression. Biol Psychiatry. 2012; 72(11): 943-949.

107. Platzer M, Dalkner N, Fellendorf FT, et al. Tryptophan breakdown and cognition in bipolar disorder. Psychoneuroendocrinology. 2017; 81: 144-150.

108. Meyer U. Developmental neuroinflammation and schizophrenia. Prog Neuropsychopharmacol Biol Psychiatry. 2013; 42: 20-34.

109. Misiak B, Stanczykiewicz B, Kotowicz K, Rybakowski JK, Samochowiec J, Frydecka D. Cytokines and C-reactive protein alterations with respect to cognitive impairment in schizophrenia and bipolar disorder: a systematic review. Schizophr Res. 2018; 192: 16-29.

110. Frydecka D, Misiak B, Pawlak-Adamska E, et al. Interleukin-6: the missing element of the neurocognitive deterioration in schizophrenia? The focus on genetic underpinnings, cognitive impairment and clinical manifestation. Eur Arch Psychiatry Clin Neurosci. 2015; 265(6): 449-459.

111. Fillman SG, Weickert TW, Lenroot RK, et al. Elevated periphera cytokines characterize a subgroup of people with schizophrenia displaying poor verbal fluency and reduced Broca's area volume. Mol Psychiatry. 2016; 21(8): 1090-1098.

112. Xiu MH, Tian L, Chen S, et al. Contribution of IL-10 and its -592 A/C polymorphism to cognitive functions in first-episode drugnaive schizophrenia. Brain Behav Immun. 2016; 57: 116-124.

113. Xiu MH, Yang GG, Tan YL, et al. Decreased interleukin-10 serum levels in first-episode drug-naive schizophrenia: relationship to psychopathology. Schizophr Res. 2014; 156(1): 9-14.

114. Fischer EK, Drago A. A molecular pathway analysis stresses the role of inflammation and oxidative stress towards cognition in schizophrenia. J Neural Transm (Vienna). 2017; 124(7): 765-774.

115. Müller N, Riedel M, Schwarz MJ, Engel RR. Clinical effects of COX-2 inhibitors on cognition in schizophrenia. Eur Arch Psychiatry Clin Neurosci. 2005; 255(2): 149-151.

116. Levkovitz Y, Mendlovich S, Riwkes S, et al. A double-blind, randomized study of minocycline for the treatment of negative and cognitive symptoms in early-phase schizophrenia. J Clin Psychiatry. 2010; 71(2): 138-149.

117. Monji A, Kato T, Kanba S. Cytokines and schizophrenia: microglia hypothesis of schizophrenia. Psychiatry Clin Neurosci. 2009; 63(3): 257-265.

118. Laskaris LE, Di Biase MA, Everall I, et al. Microglial activation and progressive brain changes in schizophrenia. Br J Pharmacol. 2016; 173(4): 666-680.

119. Linderholm KR, Skogh E, Olsson SK, et al. Increased levels of kynurenine and kynurenic acid in the CSF of patients with schizophrenia. Schizophr Bull. 2012; 38(3): 426-432.

120. Müller N. Inflammation and the glutamate system in schizophrenia: implications for therapeutic targets and drug development. Expert Opin Ther Targets. 2008; 12(12): 1497-1507. 
121. Passos IC, Vasconcelos-Moreno MP, Costa LG, et al. Inflammatory markers in post-traumatic stress disorder: a systematic review, meta-analysis, and meta-regression. Lancet Psychiatry. 2015; 2 (11): 1002-1012.

122. Eraly SA, Nievergelt CM, Maihofer AX, et al. Assessment of plasma C-reactive protein as a biomarker of posttraumatic stress disorder risk. JAMA Psychiatry. 2014; 71(4): 423-431.

123. Miller K, Driscoll D, Smith LM, Ramaswamy S. The role of inflammation in late-life post-traumatic stress disorder. Mil Med. 2017; 182(11): e1815-e1818.

124. Monsey MS, Gerhard DM, Boyle LM, Briones MA, Seligsohn M, Schafe GE. A diet enriched with curcumin impairs newly acquired and reactivated fear memories. Neuropsychopharmacology. 2015; 40(5): 1278-1288.

125. O'Donovan A, Chao LL, Paulson J, et al. Altered inflammatory activity associated with reduced hippocampal volume and more severe posttraumatic stress symptoms in Gulf War veterans. Psychoneuroendocrinology. 2015; 51: 557-566.

126. Boku S, Nakagawa S, Toda H, Hishimoto A. Neural basis of major depression: beyond monoamine hypothesis. Psychiatry Clin Neurosci. 2018; 72(1): 3-12.

127. Golier JA, Caramanica K, Michaelides AC, et al. A randomized, double-blind, placebo-controlled, crossover trial of mifepristone in Gulf War veterans with chronic multisymptom illness. Psychoneuroendocrinology. 2016; 64: 22-30.

128. Müller N, Riedel M, Schwarz MJ. Psychotropic effects of COX-2 inhibitors-a possible new approach for the treatment of psychiatric disorders. Pharmacopsychiatry. 2004; 37(6): 266-269.

129. Ayorech Z, Tracy DK, Baumeister D, Giaroli G. Taking the fuel out of the fire: evidence for the use of anti-inflammatory agents in the treatment of bipolar disorders. J Affect Disord. 2015; 174: 467-478.

130. Kohler O, Benros ME, Nordentoft M, et al. Effect of antiinflammatory treatment on depression, depressive symptoms, and adverse effects: a systematic review and meta-analysis of randomized clinical trials. JAMA Psychiatry. 2014; 71(12): 13811391.

131. Syed H, Ikram MF, Yaqinuddin A, Ahmed T. Cyclooxygenase I and II inhibitors distinctly enhance hippocampal- and cortexdependent cognitive functions in mice. Mol Med Rep. 2015; 12(5): 7649-7656.

132. Naeem S, Ikram R, Khan SS, Rao SS. NSAIDs ameliorate cognitive and motor impairment in a model of parkinsonism induced by chlorpromazine. Pak J Pharm Sci. 2017; 30(3): 801-808.

133. Vauzour D, Martinsen A, Laye S. Neuroinflammatory processes in cognitive disorders: is there a role for flavonoids and n-3 polyunsaturated fatty acids in counteracting their detrimental effects? Neurochem Int. 2015; 89: 63-74.

134. Bazinet RP, Laye S. Polyunsaturated fatty acids and their metabolites in brain function and disease. Nat Rev Neurosci. 2014; 15(12): 771-785.

135. Fourrier C, Remus-Borel J, Greenhalgh AD, et al. Docosahexaenoic acid-containing choline phospholipid modulates LPS-induced neuroinflammation in vivo and in microglia in vitro. $J$ Neuroinflammation. 2017; 14(1): 170.

136. Rey C, Nadjar A, Buaud B, et al. Resolvin D1 and E1 promote resolution of inflammation in microglial cells in vitro. Brain Behav Immun. 2016; 55: 249-259.

137. Bensalem J, Servant L, Alfos S, et al. Dietary polyphenol supplementation prevents alterations of spatial navigation in middle-aged mice. Front Behav Neurosci. 2016; 10: 9.

138. Eyre H, Baune BT. Neuroimmunological effects of physical exercise in depression. Brain Behav Immun. 2012; 26(2): 251-266.

139. Gleeson M, Bishop NC, Stensel DJ, Lindley MR, Mastana SS, Nimmo MA. The anti-inflammatory effects of exercise: mechanisms and implications for the prevention and treatment of disease. Nat Rev Immunol. 2011; 11(9): 607-615.

140. Jahshan C, Rassovsky Y, Green MF. Enhancing neuroplasticity to augment cognitive remediation in schizophrenia. Front Psychiatry. 2017; 8: 191.

141. Mittal VA, Vargas T, Osborne KJ, et al. Exercise treatments for psychosis: a review. Curr Treat Options Psychiatry. 2017; 4(2): 152-166.

142. Greer TL, Furman JL, Trivedi MH. Evaluation of the benefits of exercise on cognition in major depressive disorder. Gen Hosp Psychiatry. 2017; 49: 19-25.

143. Workman ER, Niere F, Raab-Graham KF. Engaging homeostatic plasticity to treat depression. Mol Psychiatry. 2018; 23: 26-35.

144. Ren Z, Pribiag H, Jefferson SJ, et al. Bidirectional homeostatic regulation of a depression-related brain state by gammaaminobutyric acidergic deficits and ketamine treatment. Biol Psychiatry. 2016; 80(6): 457-468.

145. Eisch AJ, Petrik D. Depression and hippocampal neurogenesis: a road to remission? Science. $2012 ; \mathbf{3 3 8}(6103)$ : 72-75.

146. Fischer S, Strawbridge R, Vives AH, Cleare AJ. Cortisol as a predictor of psychological therapy response in depressive disorders: systematic review and meta-analysis. Br J Psychiatry. 2017; 210(2): 105-109.

147. Zorn JV, Schur RR, Boks MP, Kahn RS, Joels M, Vinkers CH. Cortisol stress reactivity across psychiatric disorders: a systematic review and meta-analysis. Psychoneuroendocrinology. 2017; 77: 25-36.

148. Ribolsi M, Lisi G, Ponzo V, et al. Left hemispheric breakdown of LTP-like cortico-cortical plasticity in schizophrenic patients. Clin Neurophysiol. 2017; 128(10): 2037-2042.

149. Strube W, Bunse T, Nitsche MA, Palm U, Falkai P, Hasan A. Differential response to anodal tDCS and PAS is indicative of impaired focal LTP-like plasticity in schizophrenia. Behav Brain Res. 2016; 311: 46-53.

150. Wang HX, Gao WJ. Prolonged exposure to NMDAR antagonist induces cell-type specific changes of glutamatergic receptors in rat prefrontal cortex. Neuropharmacology. 2012; 62(4): 1808-1822.

151. Inta D, Meyer-Lindenberg A, Gass P. Alterations in postnatal neurogenesis and dopamine dysregulation in schizophrenia: a hypothesis. Schizophr Bull. 2011; 37(4): 674-680.

152. Kheirollahi M, Kazemi E, Ashouri S. Brain-derived neurotrophic factor gene Val66Met polymorphism and risk of schizophrenia: a meta-analysis of case-control studies. Cell Mol Neurobiol. 2016; 36 (1): 1-10.

153. Fernandes BS, Steiner J, Berk M, et al. Peripheral brain-derived neurotrophic factor in schizophrenia and the role of antipsychotics: meta-analysis and implications. Mol Psychiatry. 2015; 20(9): 1108-1119.

154. Sanz-Garcia A, Knafo S, Pereda-Perez I, Esteban JA, Venero C, Armario A. Administration of the TrkB receptor agonist 7,8dihydroxyflavone prevents traumatic stress-induced spatial memory deficits and changes in synaptic plasticity. Hippocampus. 2016; 26(9): 1179-1188.

155. Li X, Han F, Liu D, Shi Y. Changes of Bax, Bcl-2 and apoptosis in hippocampus in the rat model of post-traumatic stress disorder. Neurol Res. 2010; 32(6): 579-586.

156. Burgdorf J, Kroes RA, Zhang XL, et al. Rapastinel (GLYX-13) has therapeutic potential for the treatment of post-traumatic stress disorder: characterization of a NMDA receptor-mediated metaplasticity process in the medial prefrontal cortex of rats. Behav Brain Res. 2015; 294: 177-185.

157. Andrews JA, Neises KD. Cells, biomarkers, and post-traumatic stress disorder: evidence for peripheral involvement in a central disease. J Neurochem. 2012; 120(1): 26-36. 
158. Kheirbek MA, Klemenhagen KC, Sahay A, Hen R. Neurogenesis and generalization: a new approach to stratify and treat anxiety disorders. Nat Neurosci. 2012; 15(12): 1613-1620.

159. Gao J, Chen C, Liu Y, et al. Lycium barbarum polysaccharide improves traumatic cognition via reversing imbalance of apoptosis/regeneration in hippocampal neurons after stress. Life Sci. 2015; 121: 124-134.

160. Martinotti G, Sepede G, Brunetti M, et al. BDNF concentration and impulsiveness level in post-traumatic stress disorder. Psychiatry Res. 2015; 229(3): 814-818.

161. Stratta P, Sanita P, Bonanni RL, et al. Clinical correlates of plasma brain-derived neurotrophic factor in post-traumatic stress disorder spectrum after a natural disaster. Psychiatry Res. 2016; 244: 165170.

162. Angelucci F, Ricci V, Gelfo F, et al. BDNF serum levels in subjects developing or not post-traumatic stress disorder after trauma exposure. Brain Cogn. 2014; 84(1): 118-122.

163. Morris MC, Compas BE, Garber J. Relations among posttraumatic stress disorder, comorbid major depression, and HPA function: a systematic review and meta-analysis. Clin Psychol Rev. 2012; 32 (4): 301-315.

164. Klaassens ER, Giltay EJ, Cuijpers P, van Veen T, Zitman FG. Adulthood trauma and HPA-axis functioning in healthy subjects and PTSD patients: a meta-analysis. Psychoneuroendocrinology. $2012 ; 37(3): 317-331$. 